\title{
Supercritical Carbon Dioxide Sterilization
}

National Cancer Institute

\section{Source}

National Cancer Institute. Supercritical Carbon Dioxide Sterilization. NCI Thesaurus. Code C107417.

A sterilization process that uses supercritical carbon dioxide to kill microorganisms. 